Proceedings of SALT 25: 57-81, 2015

\title{
Incremental and predictive interpretation: Experimental evidence and possible accounts *
}

\author{
Adrian Brasoveanu \\ University of California Santa Cruz
}

\author{
Jakub Dotlačil \\ University of Groningen
}

\begin{abstract}
The main question we investigate is whether meaning representations of the kind that are pervasive in formal semantics are built up incrementally and predictively when language is used in real time, in much the same way that the realtime construction of syntactic representations has been argued to be. The interaction of presupposition resolution with conjunctions vs. conditionals with a sentence-final antecedent promises to provide us with the right kind of evidence. Consider the following 'cataphoric' example and the contrast between AND and IF: Tina will have coffee with Alex again $\mathrm{AND} / \mathrm{IF}$ she had coffee with him at the local café. We expect the second clause to be more difficult after AND than after IF: the conjunction AND signals that an antecedent that could resolve the again presupposition is unlikely to come after this point (the second conjunct is interpreted relative to the context provided by the first conjunct), while the conditional IF leaves open the possibility that a suitable resolution for the again presupposition is forthcoming (the first clause is interpreted relative to the context provided by the second clause). We present experimental evidence supporting these predictions and discuss two approaches to analyze this kind of data.
\end{abstract}

Keywords: incremental and predictive interpretation, cataphora, presupposition, dynamic semantics, ACT-R

\section{Introduction}

Given the detailed, theoretically rich, and empirically robust formal semantics theories that are currently available, we can ask how speakers/hearers build meaning representations when language is used in real time. Thus, the broad question behind the investigation reported in this paper is this: what is the nature of real-time semantic

* We are grateful to Stavroula Alexandropoulou, Pranav Anand, Nate Arnett, Sandy Chung, Donka Farkas, Hans Kamp, Bert Le Bruyn, Jim McCloskey, Rick Nouwen, Paul Portner, Matt Wagers, the UCSC S-circle audience (April 2015), the Georgetown Linguistics Speaker Series audience (April 2015), the SALT 25 audience (May 25), the EliTU audience (June 2015) and the participants in the UCSC spring 2015 graduate seminar Computing Dynamic Meanings for comments and discussion. Jakub Dotlačil was supported by the NWO Veni Grant 275.80.005. The usual disclaimers apply.

(C)2015 Brasoveanu and Dotlačil 
interpretation? In particular, do we build meaning representations (of the kind that are developed and deployed in formal semantics) incrementally and predictively when language is used in real time - in much the same way that the real-time construction of syntactic representations has been argued to be incremental and predictive (Steedman 2001; Lewis \& Vasishth 2005; Lau 2009; Hale 2011 among many others)?

At a very general level, our investigation is structured around the competenceperformance distinction formulated in Chomsky 1965: 3-4 which, in its most basic form, is inherited from structuralist linguistics (e.g., the langue-parole distinction in de Saussure 1916). And we take it as a given that performance models have to be based on and incorporate generative grammars (Chomsky 1965: 15). But we take the ultimate goal of semantic theory to be the development of a formally explicit theory of the cognitive behavior and cognitive mechanisms involved in natural language interpretation. From this perspective, the competence-performance distinction and the usual focus on 'competence' theories is a methodologically useful way to start the investigation, but it cannot be the end. As Clark \& Malt (1984: 191-192) put it, devising competence theories "without considering the role they would play in models of speech production and understanding [is] as if one division of General Motors were designing automobile engines without consulting the division designing the chassis into which these engines would be installed." We argue in this paper that semantic theories are ultimately incomplete if no attempt is made to embed them into mathematically explicit theories of how formal semantics representations are processed in real time.

We are interested in the incremental processing of semantic representations, and this presents us with a major empirical challenge: what phenomena can tease apart the syntactic and semantic components of the interpretation process? The pervasive aspects of meaning composition that are syntax-based cannot provide an unambiguous window into the nature of semantic representation building. The incremental and predictive nature of real-time compositional interpretation could be primarily or exclusively due to our processing strategies for building syntactic representations.

There is a significant amount of work in psycholinguistics on incremental interpretation (Hagoort, Hald, Bastiaansen \& Petersson 2004; Pickering, McElree, Frisson, Chen \& Traxler 2006 among many others), but this research usually focuses on the processing of lexical semantic and syntactic representations, and the incremental integration of world knowledge into the language interpretation process. The processing of logical representations of the kind formal semanticists are interested in is much less studied. Similarly, there is a significant amount of work in natural language processing / understanding on incremental interpretation (Poesio 1994; Hough, Kennington, Schlangen \& Ginzburg 2015 among many others), but this 
Incremental and predictive interpretation

research usually discusses it from a formal and implementation perspective, and focuses much less on the cognitive aspects of processing semantic representations (the research in Steedman 2001 and related work is a notable exception).

In this paper, we will consider novel evidence to argue for the incremental nature of formal semantic representations: the interaction of $(i)$ presupposition resolution and (ii) conjunctions vs. conditionals with a sentence-final antecedent. Consider the contrast between AND and IF in the example below, where the presupposition trigger again is cataphoric:

(1) Tina will have coffee with Alex again AND/IF she had coffee with him at the local café.

Assume the construction of semantic representations is incremental, i.e., the interpreter processes if as soon as it is encountered, and predictive, i.e., the interpreter builds a semantic evaluation structure such that the upcoming if-clause provides (some of) the interpretation context for the previously processed matrix clause. Then we expect to see a facilitation/speed-up in the second clause she had coffee with him after IF compared to AND; and this is what our experimental results actually show.

In more detail: we expect the second clause in (1) to be more difficult after AND than after IF because AND signals that a potential antecedent for the again presupposition is unlikely to come after this point - since the second conjunct is interpreted relative to the context provided by the first conjunct. In contrast, IF leaves open the possibility that a suitable resolution for the again presupposition is forthcoming since the first clause is interpreted relative to the context provided by the second clause. This possibility allows interpreters to make better predictions about the content of the clause coming after IF, which should ease its processing. Crucially, our expectations triggered by the interaction of the presupposition trigger again, and the operators AND vs. IF are semantically driven; nothing in the syntax of conjunction vs. if-adjunction could make a successful presupposition resolution more or less likely.

The paper is structured as follows. We first present our experimental results. The full experimental setup for our self-paced reading experiments turns out to be more complicated than initially expected. We discuss why that is and identify methodological issues related to using self-paced reading to study this kind of semantic issues. To this end, we begin in Section $\$ 2$ with a self-paced reading experiment that employed pronominal anaphora/cataphora (rather than cataphoric presupposition resolution) to investigate the nature of real-time semantic interpretation. The results of that experiment were suggestive, but not strong enough. This prompted us to run a follow-up experiment, reported in Section $\S 3$, in which we made the experimental design more complex, and also used presuppositional again to elicit stronger responses. In Section $\S 4$, we outline two different types of accounts that 
capture the incremental and predictive nature of real-time construction of meaning representations $(i)$ in the semantics (Incremental Dynamic Predicate Logic) or (ii) in the processor (an ACT-R based left-corner style DRT parser). We end with a brief set of conclusions (Section §5).

\section{Experiment 1: anaphora vs. cataphora in conjunctions vs. conditionals}

Elbourne (2009: 1) defines donkey cataphora as "a configuration in which a pronoun precedes and depends for its interpretation on an indefinite that does not c-command it." Some cataphora examples with conditionals are provided below, both with sentence-initial, (2)-(6), and with sentence-final if-clauses, (7).

(2) If it is overcooked, a hamburger doesn't taste good. (Chierchia 1995: 129)

(3) If she finds it spectacular, a photographer takes many pictures of a landscape. (Chierchia 1995: 130)

(4) If it enters his territory, a pirate usually attacks a ship. (Chierchia 1995: 130)

(5) If it spots a mouse, a cat attacks it. (Chierchia 1995: 130)

(6) If a foreigner asks him for directions, a person from Milan replies to him with courtesy. (Chierchia 1995: 130)

(7) John won't eat it if a hamburger is overcooked. (Elbourne 2009: 3)

Certain configurations are not acceptable (Elbourne 2009: 2), e.g., (8c) below, presumably due to Principle $\mathrm{C}$ violations. Antecedents are marked with a superscript, and the corresponding anaphors/cataphors are marked with a subscript.
a. John ${ }^{i}$ is upset if he ${ }_{i}$ sees a donkey.
b. If $\mathrm{John}^{i}$ sees a donkey, he ${ }_{i}$ is upset.
c. ${ }^{*} \mathrm{He}_{i}$ is upset if $\mathrm{John}^{i}$ sees a donkey.

The contrast between (8b) and (8c), as well as the fact that Principle $\mathrm{C}$ is not violated if cataphoric pronouns appear in object position - see (7) above and (9) below, provide evidence that a sentence-final if-clause is adjoined lower than the matrix-clause subject but higher than the object (let's say VP-adjoined for concreteness). In contrast, a sentence-initial if-clause is adjoined higher than the matrix-clause subject (again, let's say it is CP-adjoined for concreteness).

(9) Bill visits her if $_{i}$ Mary $_{i}$ is sick.

That is, the syntax of conditionals with sentence-initial vs. sentence-final if clauses is basically the one in Figure 1. Other arguments for these two syntactic structures are provided by VP ellipsis, as in (10), and VP topicalization, as in (11); see Bhatt \& Pancheva (2006) for more discussion. 
Incremental and predictive interpretation

(8b)

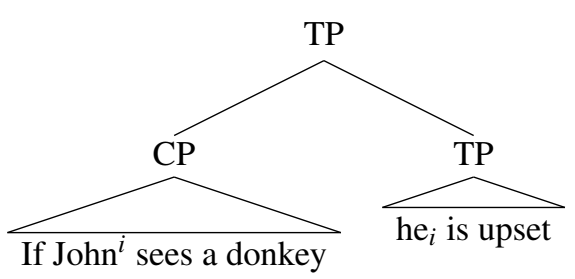

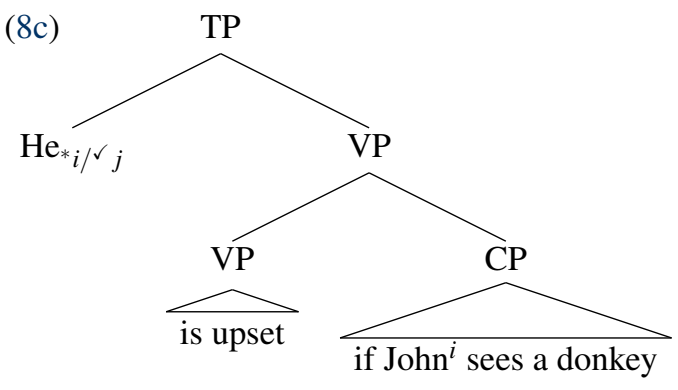

Figure 1 The syntactic structure of conditionals with initial vs. final if-clauses

(10) I will leave if you do, and John will [leave if you de] too / do so too.

(11) I told Peter to take the dog out if it rains, and [take the dog out if it rains] he will. (Iatridou 1991: 12)

Thus, there is no 'ordinary' syntax-mediated binding from a c-commanding position for direct object (DO) donkey cataphora in conditionals with sentence-final if clauses; i.e., this is a 'true' example of donkey cataphora that we can use.

Experiment 1 tested donkey anaphora and cataphora in a $2 \times 2$ design, exemplified here:

Experiment 1: AND/IF $\times$ DO ANAPHORA/CATAPHORA

a. An electrician examined a radio for several minutes AND his helpful colleague held it that whole time.

AND \& ANAPHORA

b. An electrician examined a radio for several minutes IF his helpful colleague held it that whole time.

IF \& ANAPHORA

c. An electrician examined it for several minutes AND his helpful colleague held the radio that whole time.

AND \& CATAPHORA

d. An electrician examined it for several minutes IF his helpful colleague held the radio that whole time.

IF \& CATAPHORA

Kazanina, Lau, Lieberman, Yoshida \& Phillips (2007) used an on-line reading methodology (self-paced reading) to show that a cataphoric pronoun triggers an active search for an antecedent in the following material, and that this search takes into account structural constraints (Principle C) from an early stage. ${ }^{1}$ Kazanina et al. (2007) take the temporal priority of syntactic information as evidence for the incremental and predictive nature of syntactic constraints. The question we want

1 That is, cataphoric dependencies are processed with a syntactically constrained search mechanism similar to the mechanism used for processing long-distance wh-dependencies (Stowe 1986; Traxler \& Pickering 1996; Wagers \& Phillips 2009 a.o.). 
to investigate in Experiment 1 can therefore be further specified as follows: is this active search mechanism also semantically constrained?

Method. We tested the aforementioned question using self-paced reading (Just, Carpenter \& Woolley 1982) with a moving window. ${ }^{2}$ The experiment consisted of 28 items and 107 fillers. The fillers were monoclausal and multiclausal, including conditionals, conjunctions, when-clauses, relative clauses, quantifiers, adverbs etc. 62 native speakers of English (UCSC undergraduate students) completed the experiment online for course (extra-)credit on a UCSC hosted installation of Alex Drummond's IBEX platform (http://code.google.com/p/webspr/). Each item was passed through 4 conditions, and 4 lists were generated following a Latin square design (each list had the following structure: every item appeared once, with 7 items per condition; the items were rotated through the 4 conditions across the 4 lists). The participants were rotated through the 4 lists; every participant responded to 135 stimuli (28 items +107 fillers), the order of which was randomized for every participant (any two items separated by at least one filler). There were 54 comprehension questions with correct/incorrect answers, 9 after experimental items; 5 outlier participants were excluded because of their low accuracy $(\leq 80 \%)$.

Predictions. First, the regular anaphora cases provide the baseline conditions. Assuming a deep enough incremental and predictive interpretation, we expect the second clause in these conditions to be more difficult after IF than after AND because of extra cognitive load coming from two sources.

The first source of difficulty is the semantics of conditionals vs. conjunctions. For conditionals, we generate a hypothetical intermediate interpretation context satisfying the antecedent, and we evaluate the consequent relative to this context. That is, we need to maintain both the actual, global interpretation context and the intermediate, antecedent-satisfying context, to complete the interpretation of conditionals. There is no similar cognitive load for conjunctions.

The second source of difficulty is specific to conditionals with a sentencefinal if-clause. When such conditionals are incrementally interpreted, the matrix clause needs to be semantically reanalyzed: the matrix clause is initially analyzed/interpreted relative to the global context, but when if is reached, the matrix clause has to be reinterpreted relative to the intermediate, antecedent-satisfying context. Again, there is no such difficulty for conjunctions: the first conjunct is simply

2 In this task, each stimulus is first presented on a computer screen with all words hidden and participants have to press the Space bar to reveal the first word. Afterwards, each time the Space bar is pressed, the next word is revealed and the previous one is hidden. At the end of any stimulus, pressing the Space bar reveals the follow-up question or the next stimulus. 
Incremental and predictive interpretation

interpreted relative to the global context, and the second conjunct is interpreted relative to the context that is the result of the update contributed by the first-conjunct.

For the cataphora (non-baseline cases), we expect a cognitive load reversal. AND signals that no suitable antecedent for the cataphor is forthcoming since the second clause is interpreted relative to the context provided/updated by the first clause. In contrast, IF triggers semantic reanalysis and leaves open the possibility that a suitable antecedent for the cataphor is forthcoming since the first clause is interpreted relative to the context provided/updated by the second clause. This expectation (and the fact that it is confirmed) should speed up the processing. So we expect to see a speed-up in the IF \& CATAPHORA cases, i.e., a negative IF $\times$ CATAPHORA interaction.

The predictions were only partially confirmed: baseline IF was indeed harder (statistically significant) but the IF $\times$ CATAPHORA interaction, while negative, did not reach significance.

Results. The regions of interest (ROIs) were primarily $(i)$ the post-connective ROIs his helpful colleague, and secondarily (ii) the post-resolution ROIs that whole; see (13) below. The post-resolution ROIs are of merely secondary interest because of the asymmetry between the anaphora conditions, which contain an indefinite ( a radio), and the cataphora conditions, which contain an definite (the radio). We introduced a definite in the cataphora conditions because we wanted (some of) the items to sound more natural overall; this doesn't affect the ROIs of primary interest, which precede the definite. The mean $\log$ reading times (log RTs) for the 5 ROIs are plotted in Figure 2 (plots created with R and ggplot2; R Core Team 2014; Wickham 2009).

An electrician examined a radio/it for several minutes and/if his helpful colleague held it/the radio that whole time.

We analyzed the data using linear mixed-effects models. ${ }^{3}$ The response was the log-transformed readings times (log RTs) for the ROIs (log-transformed to mitigate right-skewness of RTs); we also analyzed the residualized log RTs (residualized for word length and word position, following Trueswell, Tanenhaus \& Garnsey 1994), but the pattern of results did not change, so we report the more easily intelligible models with raw log RT responses. Predictors (fixed effects): main effects of CONNECTIVE and ANA/CATAPHORA, and their interaction; the levels of the CONNECTIVE factor: AND (reference level) vs. IF; the levels of the ANA/CATAPHORA factor: ANAPHORA (reference level) vs. CATAPHORA. We included crossed random effects for subjects and items; in particular, we report models with the maximal

3 estimated with R, Ime4, ImerTest (R Core Team 2014; Bates, Maechler, Bolker \& Walker 2014; Kuznetsova, Bruun Brockhoff \& Haubo Bojesen Christensen 2014) 


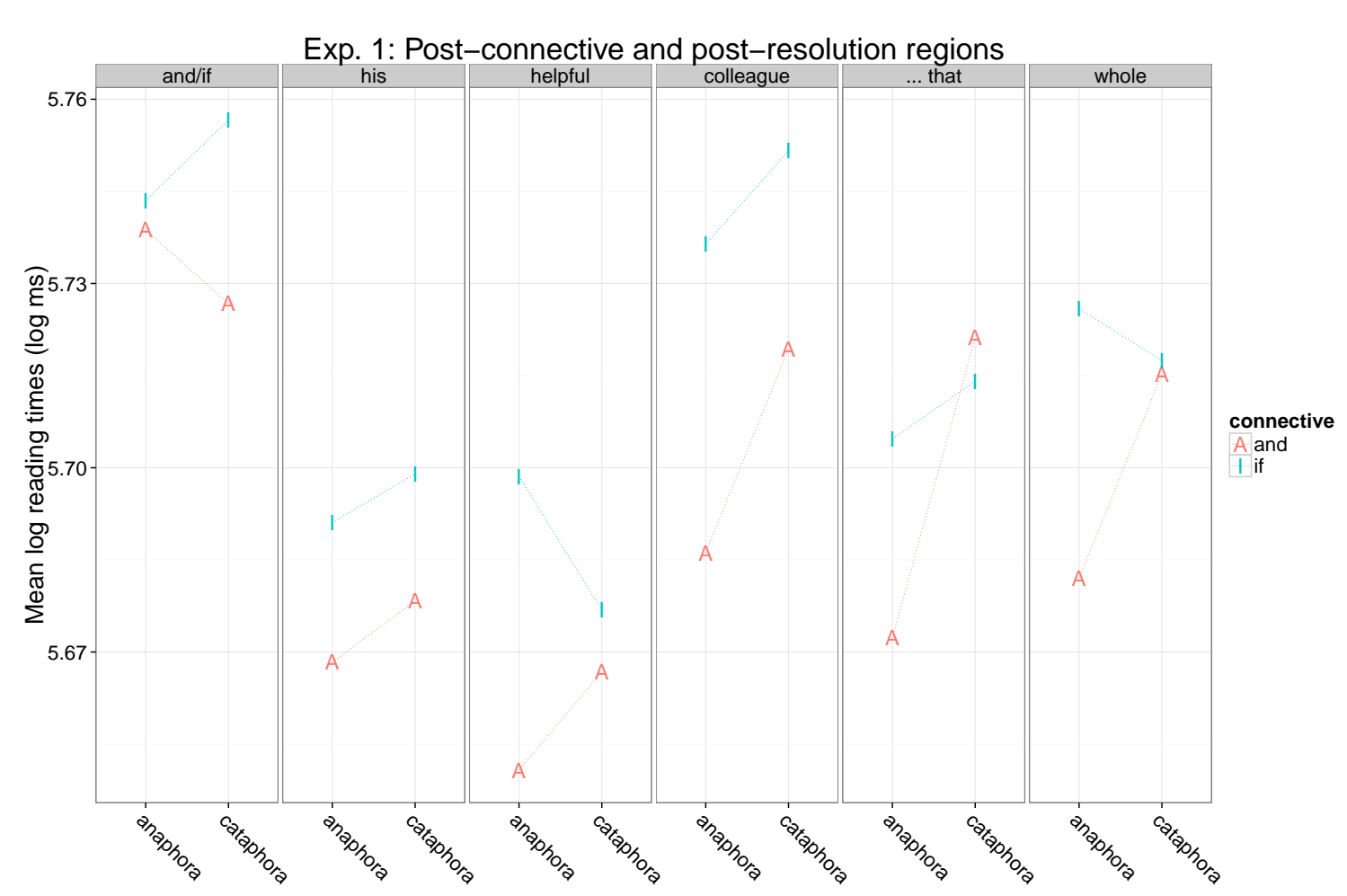

Figure 2 Experiment 1: mean log RTs for the five regions of interest (ROIs)

random effect structure that converged (Barr, Levy, Scheepers \& Tily 2013), usually subject and item random intercepts, and subject and item random slopes for at least one of the two main effects. The maximum likelihood estimates (MLEs) and associated standard errors (SEs) and $p$-values are provided in (14) and (15) below. Significant and nearly significant effects $(p<.1)$ are boldfaced.

(14)

\begin{tabular}{l||rrr|rrr|rrr}
\multicolumn{1}{l||}{} & \multicolumn{2}{c|}{ his } & \multicolumn{3}{c|}{ helpful } & \multicolumn{3}{c}{ colleague } \\
& MLE & SE & $p$ & MLE & SE & $p$ & MLE & SE & $p$ \\
\hline \hline IF & 0.02 & 0.02 & 0.3 & $\mathbf{0 . 0 5}$ & $\mathbf{0 . 0 2}$ & $\mathbf{0 . 0 4}$ & $\mathbf{0 . 0 5}$ & $\mathbf{0 . 0 2}$ & $\mathbf{0 . 0 4}$ \\
CATA & 0.01 & 0.02 & 0.7 & 0.02 & 0.02 & 0.4 & 0.03 & 0.02 & 0.16 \\
IF $\times$ CATA & -0.003 & 0.03 & 0.9 & -0.04 & 0.03 & 0.15 & -0.02 & 0.03 & 0.6
\end{tabular}

$(15)$

\begin{tabular}{l||rrr|rrr}
\multicolumn{1}{l||}{} & \multicolumn{3}{c|}{ that } & \multicolumn{3}{c}{ whole } \\
& MLE & SE & $p$ & MLE & SE & $p$ \\
\hline \hline IF & 0.03 & 0.02 & 0.20 & 0.03 & 0.02 & 0.24 \\
CATA & $\mathbf{0 . 0 4}$ & $\mathbf{0 . 0 2}$ & $\mathbf{0 . 0 7}$ & 0.03 & 0.02 & 0.18 \\
IF $\times$ CATA & -0.03 & 0.03 & 0.26 & -0.03 & 0.03 & 0.43
\end{tabular}


Incremental and predictive interpretation

Generalizations and their consequences. Baseline IF (i.e., IF \& ANAPHORA) is more difficult than baseline AND (i.e., AND \& ANAPHORA). This is compatible with the hypothesis that to interpret conditionals, we need to maintain both the actual, global interpretation context and the intermediate, antecedent-satisfying context, and/or with the hypothesis that the matrix clause is reanalyzed in conditionals with a final if-clause: it is initially interpreted relative to the global context until if is reached, then it is reinterpreted at that point relative to the intermediate, antecedentsatisfying context.

CATAPHORA seems to be more difficult than ANAPHORA for AND, but the effect never reaches significance (close to significant in the first ROI after cataphora is resolved). Maybe the AND \& CATAPHORA condition is simply too hard, so readers stop trying to fully comprehend the sentence and speed up. If so, this will obscure the IF $\times$ CATA interaction - and this is exactly what seems to be happening: there is a negative interaction between IF and CATAPHORA in all ROIs, i.e., IF seems to facilitate CATAPHORA (as expected if semantic evaluation is incremental and predictive), but this effect is not significant.

The consistent negative interaction is promising, so we will try to elicit it with a hard presupposition trigger like again (Abusch 2010; Schwarz 2014 a.o.), which might have a larger effect. We will also add a (mis)match manipulation to control for readers speeding up through conditions that are too hard.

\section{Experiment 2: cataphoric presuppositions in conjunctions vs. conditionals}

The experiment was a $2 \times 2 \times 2$ design, exemplified below on one item. The match and mismatch (new manipulation compared to Experiment 1) was achieved by matching and mismatching verbs in the second clause.

(16) Experiment 2: (MIS)MATCH $\times$ AND/IF $\times$ NOTHING/CATAPHORA

a. Jeffrey will argue with Danielle AND he argued with her in the courtyard last night.

MATCH \& AND \& NOTHING

b. Jeffrey will argue with Danielle IF he argued with her in the courtyard last night.

MATCH \& IF \& NOTHING

c. Jeffrey will argue with Danielle again AND he argued with her in the courtyard last night.

MATCH \& AND \& CATAPHORA

d. Jeffrey will argue with Danielle again IF he argued with her in the courtyard last night.

MATCH \& IF \& CATAPHORA

e. Jeffrey will argue with Danielle AND he played with her in the courtyard last night.

MISMATCH \& AND \& NOTHING 
f. Jeffrey will argue with Danielle IF he played with her in the courtyard last night.

MISMATCH \& IF \& NOTHING

g. Jeffrey will argue with Danielle again AND he played with her in the courtyard last night.

MISMATCH \& AND \& CATAPHORA

h. Jeffrey will argue with Danielle again IF he played with her in the courtyard last night.

MISMATCH \& IF \& CATAPHORA

Method. The method was similar to Exp. 1, with some differences: we still used self-paced reading with a moving window, but each stimulus ended with an acceptability judgment on a 5-point Likert scale, from 1 (very bad) to 5 (very good). The acceptability judgment was elicited on a new screen after every item or filler. Every experimental item was followed by a comprehension question. Each of the 8 conditions was tested 4 times (32 items total; one item had a typo, discarded from all subsequent analyses). There were 70 fillers (monoclausal and multiclausal, conditionals, conjunctions, when-clauses, relative clauses, quantifiers, adverbs, etc.).

32 native speakers of English participated (UCSC undergraduate students); they completed the experiment online for course (extra-)credit on a UCSC hosted installation of the IBEX platform. Each item was passed through all 8 conditions, and 8 lists were generated following a Latin square design (in each list, every item appeared once, with 4 items per conditions; the items were rotated through conditions across the 8 lists). The participants were rotated through the 8 lists; every participant responded to 102 stimuli (32 items +70 fillers), the order of which was randomized for every participant (any two items separated by at least one filler).

There were fillers that were both acceptable (Bob ate his burger and he rented something to watch, but he didn't say what) and unacceptable (Willem visited Paris because Sarah visited Amsterdam too). All participants exhibited the expected difference in acceptability ratings between these 2 types of fillers.

There were 72 comprehension questions with correct/incorrect answers, 32 after experimental items. The accuracy for all participants was above $80 \%$.

Predictions. The $4 \mathrm{MATCH}$ conditions are parallel to the 4 conditions in Exp. 1, so we make similar predictions for them. The MISMATCH conditions allow us to control for readers speeding up through conditions that are too hard. For example, if AND \& CATAPHORA is too hard and readers stop trying to fully comprehend the sentence, we won't see a difference between the MATCH and MISMATCH cases. Correspondingly, if readers interpret IF \& CATAPHORA deeply enough, and incrementally and predictively, we expect a slow down for the MISMATCH condition. That is, we expect a positive 3 -way interaction for IF $\times$ CATAPHORA $\times$ MISMATCH: readers expect a suitable antecedent for the again presupposition, and the antecedent 
Incremental and predictive interpretation

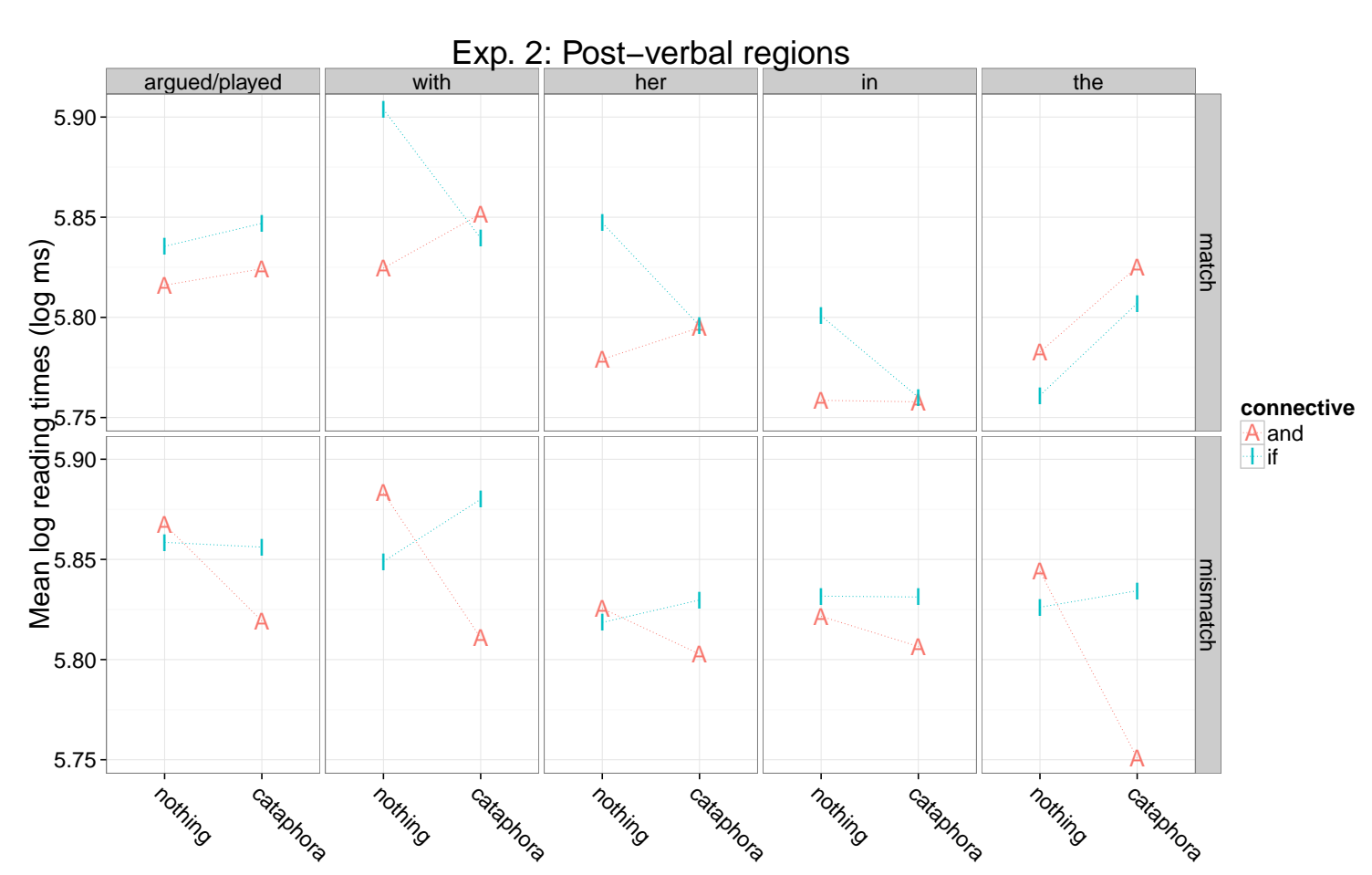

Figure 3 Experiment 2: mean $\log$ RTs for the four regions of interest (ROIs)

is not provided in the MISMATCH cases, which will lead to processing difficulties (see Hale 2001 and Levy 2008, among others, for similar arguments with respect to syntactic comprehension).

Results. The ROIs for Exp. 2 are the words following the verb in the second clause, i.e., just as before, the words immediately following the last experimental manipulation, which is (MIS)MATCH in Exp. 2. We examine only the 4 immediately post-verbal ROIs because the 5th word was the final one for some items, and the wrap-up effect associated with sentence-final words would contribute additional, possibly biasing noise.

(17) Jeffrey will argue with Danielle $\emptyset$ /again and/if he argued/played with her in the courtyard last night.

The data analysis was very similar to the one we conducted for Exp. 1. We used linear mixed-effects models, with log RTs as the response variable, and main effects of CONNECTIVE and NOTHING/CATAPHORA, MATCH/MISMATCH and their 2-way and 3-way interactions as predictors (fixed effects). The levels of the 3 factors were as follows: for CONNECTIVE, AND (reference level) vs. IF, for NOTHING/CATAPHORA: 
NOTHING (reference level) vs. CATAPHORA, for (MIS)MATCH: MATCH (reference level) vs. MISMATCH. The models also included crossed random effects for subjects and items, namely the maximal random effect structure that converged, usually subject and item random intercepts, and subject and item random slopes for at least two of the three main effects.

\begin{tabular}{l||rrr|rrr|rrr|rrr}
\multicolumn{1}{c|}{$(18)$} & \multicolumn{3}{c|}{ with } & \multicolumn{4}{c}{ her } & \multicolumn{4}{c}{ in } & \multicolumn{3}{c}{ the } \\
& MLE & SE & $p$ & MLE & SE & $p$ & MLE & SE & $p$ & MLE & SE & $p$ \\
\hline \hline CATA & 0.05 & 0.04 & 0.21 & 0.05 & 0.04 & 0.30 & 0.03 & 0.04 & 0.50 & 0.05 & 0.04 & 0.23 \\
MISMATCH & 0.05 & 0.04 & 0.25 & 0.04 & 0.04 & 0.33 & 0.06 & 0.05 & 0.19 & 0.07 & 0.05 & 0.15 \\
IF & $\mathbf{0 . 0 8}$ & $\mathbf{0 . 0 4}$ & $\mathbf{0 . 0 5 4}$ & $\mathbf{0 . 0 7}$ & $\mathbf{0 . 0 4}$ & $\mathbf{0 . 0 8 4}$ & 0.05 & 0.04 & 0.24 & -0.003 & 0.04 & 0.96 \\
CATA $\times$ MISMATCH & $\mathbf{- 0 . 1 1}$ & $\mathbf{0 . 0 6}$ & $\mathbf{0 . 0 5 6}$ & -0.05 & 0.06 & 0.42 & -0.03 & 0.06 & 0.59 & $\mathbf{- 0 . 1 4}$ & $\mathbf{0 . 0 6}$ & $\mathbf{0 . 0 3}$ \\
CATA $\times$ IF & $\mathbf{- 0 . 1 3}$ & $\mathbf{0 . 0 6}$ & $\mathbf{0 . 0 2 6}$ & $\mathbf{- 0 . 1 1}$ & $\mathbf{0 . 0 6}$ & $\mathbf{0 . 0 7 7}$ & -0.08 & 0.05 & 0.15 & -0.04 & 0.06 & 0.54 \\
MISMATCH $\times$ IF & $\mathbf{- 0 . 1 0}$ & $\mathbf{0 . 0 6}$ & $\mathbf{0 . 0 8 3}$ & -0.06 & 0.06 & 0.30 & -0.02 & 0.05 & 0.73 & -0.02 & 0.06 & 0.76 \\
CATA $\times$ MISMATCH $\times$ IF & $\mathbf{0 . 2 0}$ & $\mathbf{0 . 0 8}$ & $\mathbf{0 . 0 1 5}$ & 0.10 & 0.08 & 0.22 & 0.06 & 0.08 & 0.42 & 0.11 & 0.09 & 0.19
\end{tabular}

Generalizations and their consequences. Just as in Exp. 1, baseline IF (i.e., IF \& NOTHING \& MATCH) is more difficult than baseline AND (i.e., AND \& NOTHING \& MATCH). This is compatible with the hypothesis mentioned in Exp. 1 that conditionals are harder than conjunctions (because we need to maintain two evaluation contexts, and/or the matrix clause is semantically reanalyzed when if is reached). ${ }^{4}$

There is a significant negative interaction of MISMATCH $\times$ IF (note: again is not present here), which basically cancels out the main effect of IF. That is, conditionals with non-identical VP meanings in the antecedent and consequent clauses are processed more easily than conditionals with identical VP meanings, about as easily as conjunctions with non-identical VP meanings in the two conjuncts. The difficulties tied to conditionals with identical VP meanings are probably caused by a violation of Maximize Presupposition (Heim 1991), which requires that a presupposed VP meaning should be marked as such by again. This penalizes conditionals with matching VP meanings, while conditionals with non-identical VP meanings are not affected. Furthermore, if participants interpret incrementally and predictively, Maximize Presupposition should not affect coordinations, which corresponds to our findings.

The Maximize Presupposition constraint provides a third possible reason for the

4 Since Exp. 2 included acceptability judgments, we could check whether the effect, replicated from Exp. 1, is not due to the fact that the IF condition (Jeffrey will argue with Danielle if he argued with her in the courtyard last night) is less felicitous than the corresponding AND condition (Jeffrey will argue with Danielle and he argued with her in the courtyard last night). This is not so: the only statistically significant fixed effect was a positive main effect for IF, i.e., baseline IF is more acceptable than baseline AND. We used mixed-effects ordinal probit models to analyze the acceptability data - full fixed-effect structure (main effects + all interactions), maximal random effect structure that converged; estimated with the ordinal package (Haubo Bojesen Christensen 2013). 
Incremental and predictive interpretation

cost of baseline IF relative to baseline AND aside from the suggestions discussed before (conditionals are harder than conjunctions because we need to maintain two evaluation contexts, and/or the matrix clause is semantically reanalyzed). They all might be at work here (distinguishing between them is left for a future occasion), but Maximize Presupposition might be particularly suitable as an explanation for the Exp. 2 results: it explains the cost of IF, but it also explains the negative interaction MISMATCH $\times$ IF (which is unexpected under the hypothesis that IF on its own is costly). Furthermore, in Exp. 2, the effect of IF is observed on with and her, which makes the explanation in terms of reanalysis unlikely given the lateness of the effect. In Exp. 1, the effect of IF was detectable on the second word after if, so the reanalysis explanation is more plausible for that experiment.

There are no main effects of CATAPHORA and MISMATCH, but their 2-way interaction is negative and significant (or close to significant) in 2 out of 4 regions. Whenever (close to) significant, this interaction effectively cancels the main effects

of both MISMATCH and CATAPHORA; that is, the AND \& CATAPHORA \& MISMATCH condition is about as difficult as the reference condition AND \& NOTHING \& MATCH, which suggests that participants stopped trying to properly interpret this difficult condition (AND \& CATAPHORA \& MISMATCH) and moved on / sped up.

There is a (close to) significant negative interaction of CATAPHORA $\times$ IF in the two regions immediately following the verb (note: we are discussing MATCHING conditions). In both regions, this 2-way interaction effectively cancels out the positive main effects of CATAPHORA and IF put together. This is exactly the configuration we were looking for in Exp. 1, only it didn't reach significance there. That is, IF facilitates the processing of CATAPHORA, even though IF and CATAPHORA on their own are more difficult. This supports the hypothesis that the construction of formal semantic representations is incremental and predictive.

Finally, the statistically significant, positive 3 -way interaction CATAPHORA $\times$ IF $\times$ MISMATCH in the region immediately following the verb provides further empirical support for the hypothesis that the construction of formal semantic representations is incremental and predictive. The MISMATCH is surprising because the human interpreter expects to find a suitable antecedent for the again presupposition, and that expectation is not satisfied.

Summarizing, Experiments 1 and 2 provide coherent support for the incremental and predictive nature of the process of constructing meaning representations of the kind employed in formal semantics.

\section{Incrementality: semantics or processing?}

The main questions at this point are the following. As formal semanticists, should we account for the incremental and predictive nature of the real-time semantic 
interpretation process? And if so, how?

It is important to remember that addressing these questions is firmly rooted in the tradition of dynamic semantics. Kamp (1981: 189) begins like this:

Two conceptions of meaning have dominated formal semantics of natural language. The first of these sees meaning principally as that which determines conditions of truth. [...] According to the second conception meaning is, first and foremost, that which a language user grasps when he understands the words he hears or reads. [...] these two conceptions [...] have remained largely separated for a considerable period of time. This separation has become an obstacle to the development of semantic theory [...] The theory presented here is an attempt to remove this obstacle. It combines a definition of truth with a systematic account of semantic representations.

Thus, the implicit overarching goal for us as (cognitive) scientists studying natural language meaning is to provide a formally explicit account of natural language interpretive behavior, i.e., a mathematically explicit, unified theory of semantic/pragmatic competence and performance.

To contextualize our position and outline some possible alternatives, we consider the corresponding debate on the syntax side. Phillips \& Lewis (2013: 14) identify two reasonable positions that working linguists more or less implicitly subscribe to in practice: (i) principled extensionalism, and (ii) strategic extensionalism.

Principled extensionalism takes a grammar / grammatical theory to be merely an abstract characterization of a function whose extension is all and only the wellformed sentences of a given language (plus their denotations, if the grammar incorporates a semantic component). ${ }^{5}$ The individual components of the grammatical theory have no independent status as mental objects or processes: they are components of an abstract function, not of a more concrete description of a mental system. Note that this kind of position is beyond the reach of most empirical evidence aside from acceptability (or truth-value / entailment) judgments, since it only aims to capture the 'end products' of the grammatical system and not the way these products are actually produced / comprehended. The 'principled' part is that the extensionalist enterprise is understood as an end in itself, relevant even if lower-level characterizations of the human language system are provided (algorithmic / mechanistic, or implementation / neural level; Marr 1982). The linguist's task is to characterize what the human

5 More precisely, assume some background alphabet $\Sigma$ that consists of the lexicon / set of words ('alphabet' in the sense of formal language theory), and let $\Sigma^{*}$ be the set of all finite strings over $\Sigma$. $\Sigma^{*}$ is the domain of the function, and $\{0,1\}$ its range, so that the function is a characteristic function of the set of grammatical strings. 
Incremental and predictive interpretation

language system computes and distinguish it from how speakers actually carry out that computation, which is the psycholinguist's task.

The strategic extensionalism position takes the goal of formulating a grammatical theory to be a reasonable interim goal, but not an end in itself. The ultimate goal is to move beyond extensional description to a more detailed, mechanistic understanding of the human language system: describing an abstract function that identifies all the grammatical sentences of a language is just a first step in understanding how speakers actually comprehend / produce sentences in real time. We seek theories that capture how sentences are put together, and not just what their final form is. From this perspective, we should try to account for left-to-right structure building mechanisms, both at the syntactic and at the semantic level.

Assuming a strategic extensionalist position, the next question is: how should we account for the incremental and predictive nature of semantic interpretation? We will not settle this question here, but we will outline two distinct approaches and briefly compare them. As far as we can tell, there is a spectrum of approaches to incrementality effects, and the two extremes on that spectrum are accounting for incrementality ( $i)$ in the semantics vs. (ii) in the processor.

The first alternative is parallel to the proposal in Phillips 1996, 2003 on the syntax side. The main claim in Phillips 1996, 2003 is that syntactic structures are built left-to-right, not top-down / bottom-up, and the incremental left-to-right system is the only structure-building system that humans have ('the parser is the grammar'). Our specific proposal on the semantics side is providing a recursive definition of truth and satisfaction for first-order predicate logic that is fully incremental, building on the incremental propositional logic system in Vermeulen 1994. We dub the resulting system Incremental Dynamic Predicate Logic (IDPL).

The second alternative is parallel to the proposal in Hofmeister, Casasanto \& Sag 2013 on the syntax side, the main goal of which is to argue that "many of the findings from studies putatively supporting grammar-based interpretations of island phenomena have plausible, alternative interpretations rooted in specific, well-documented processing mechanisms" (Hofmeister et al. 2013: 44). Our specific proposal on the processing side is to devise and implement an ACT-R based (Anderson \& Lebiere 1998) left-corner style parser for DRT (Kamp \& Reyle 1993), in the style of the (Lisp) ACT-R left-corner parser for syntactic representations in Lewis \& Vasishth 2005 (but we implement ours in Python ACT-R; Stewart 2007; Stewart \& West 2007).

\subsection{Incremental Dynamic Predicate Logic (IDPL)}

Incremental Dynamic Predicate Logic (IDPL) extends the incremental semantics for Dynamic Propositional Logic (DPropL) in Vermeulen (1994) to Dynamic Predicate 
Logic (DPL; Groenendijk \& Stokhof 1991). We only give here a brief outline of how a left-to-right incremental dynamic semantics can be provided - we will just discuss the basics of DPropL. The syntax of DPropL is provided in (19). $\perp$ is the formula that is always false: $[[\perp]]=\mathbb{F}$. Negation can be defined in terms of implication and $\perp$ following the classical abbreviation $\neg \varphi:=\varphi \rightarrow \perp$; see (20). The formula $\top$ that is always true $([[T]]=\mathbb{T})$ is also defined following the classical abbreviation $\top:=\neg \perp(=\perp \rightarrow \perp)$. Finally, we define disjunction De Morgan style.

(19) The syntax of DPropL. Given a set of atomic texts $A$ (i.e., atomic prop. variables), the set of texts $T_{A}$ based on $A$ (i.e., the set of well-formed prop. formulas) is the smallest set such that

(i) $A \subseteq T_{A}$,

(ii) $\perp \in T_{A}$,

(iii) $\{$ if, then, end $\} \subseteq T_{A}$, and finally

(iv) if $\varphi, \psi \in T_{A}$, then $\varphi ; \psi \in T_{A}$ (';' symbolizes dynamic conjunction, i.e., text merging / concatenation).

(20) Abbreviations:

(i) $\neg \varphi:=$ if; $\varphi$; then; $\perp$; end,

(ii) $\top:=\neg \perp(=$ if; $\perp$; then; $\perp$; end $)$, and finally

(iii) $\varphi \vee \psi:=\neg(\neg \varphi ; \neg \psi)$.

The main notable feature of the syntax of DPropL is the fact that we use three basic expressions if, then, and end to define our notion of implication. This design choice on the syntax side is justified by the particular feature we want the semantics of DPropL to have. In particular, we want the semantics of DPropL to respect three principles (Vermeulen 1994: 244-246): (i) incrementality: we can interpret texts as we hear them; (ii) pure compositionality: 'pure' - we do not assume that a full syntactic analysis precedes interpretation (unlike standard (neo)Montagovian semantics, or even the incremental DRS construction algorithm in Kamp \& Reyle 1993); and (iii) break-in: every segment of a text should be interpretable, even if what comes after, or came before, is unknown; wherever we 'break in' in a text, interpretation should be possible. Together, $(i$-iii) entail associativity: text meanings have to form an algebra with an associative operation ('merger' / conjunction) by which the meanings can be glued together. This is OK for texts that are actually conjoined:

(21) Bob inherited a donkey $(p)$, and Jane bought it from him $(q)$, and she sold it to $\operatorname{Bill}(r)$. 
Incremental and predictive interpretation

The problem is that conditionals do not have an associative, incremental semantics. We can see that with if-clause final conditionals of the kind we examined in Experiments 1 and 2 above: incrementally, the if-clause is interpreted after the main clause, but deriving the correct interpretation for the conditional requires us to interpret the if-clause before the main clause. But the non-associativity of conditionals can be established with 'run-of-the-mill' conditionals with an initial if-clause too. Consider the text in (22): it is intuitively interpreted as in (23), not as in (24) - if $p$ is false, the text is false, not true. And a strictly incremental semantics forces the bracketing in (25), which derives the incorrect interpretation in (24).

(22) The driver was not working that night $(p)$ and if the butler was working that night $(q)$, the butler committed the murder $(r) . \quad \rightsquigarrow p$; if; $q$; then; $r$; end

Intended interpretation: $p ;($ if $; q ;$ then; $r ;$ end $)$

$$
\text { i.e., } p \wedge(q \rightarrow r)
$$

By associativity: $(p ;$ if $; q)$; then; $r$; end

$$
\text { i.e., roughly, }(p \wedge q) \rightarrow r
$$

Incremental interpretation: $((((p ;$ if $) ; q) ;$ then $) ; r)$; end

The basic solution to the problem of deriving the correct interpretation in (23) while doing composition as in $(24) /(25)$ (which preserves across-the-board associativity and strict incrementality) is memory: semantic evaluation contexts are update histories (more structured than classical FOL or standard DPL contexts). And the basic expressions if, then, and end incrementally (and associatively) update / manipulate these histories in such a way that the correct truth conditions for conditionals emerge at the end of the update sequence.

We interpret (23) as follows:

a. We first update with the main clause / first conjunct $p$ by targeting the information state $i$ that is circled in the update tree below; this update contributes new truth-conditional and anaphoric information and it is merged into the current global info state $i$ directly:

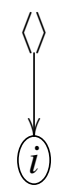

b. We then update with if; this update does not add new info (truth-conditional and / or anaphoric) content, but it manipulates the update history; in particular, it opens a new update-history branch and designates the info state $j$ at the end of that branch as the current active / updatable info state (we again indicate that $j$ is the active info state by circling it); the conditional antecedent $q$ is then merged into the info state $j$ in the regular 
way (just as $p$ was merged into the global info state $i$ ):

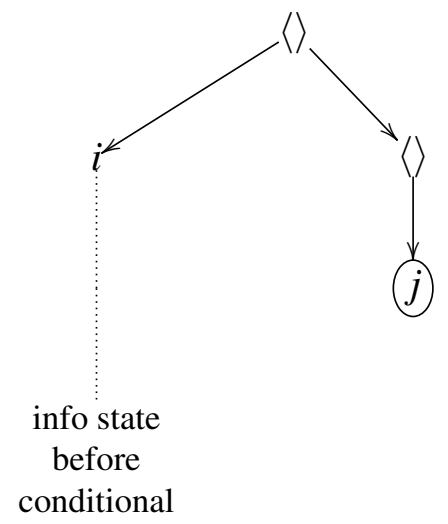

c. We then update with then; just as in the case of if, this update only manipulates the structure of the update history by opening a new update branch and designating the info state $k$ on that branch as the active one; the info state $k$ is targeted by the conditional consequent $r$ :

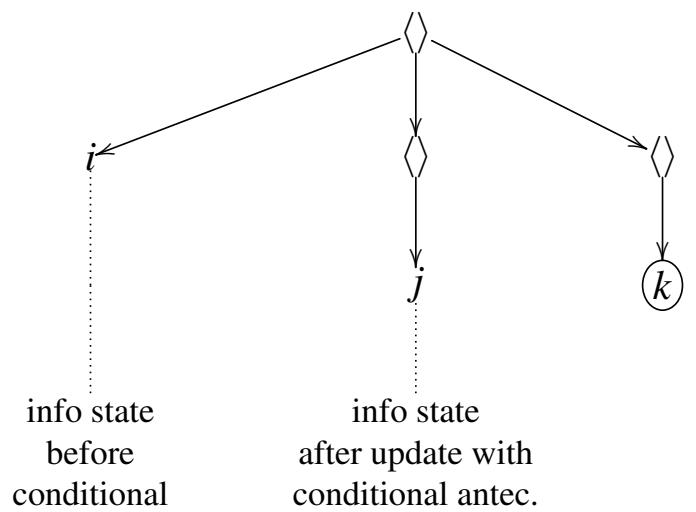

d. Finally, the update with end merges these three branches together in such a way that the correct truth conditions for the conditional are derived; the resulting info state $i \bullet(j \rightarrow k)$ becomes the new current global info state relative to which subsequent discourse is interpreted; $\bullet$ encodes the merge operation over info states, and $\rightarrow$ is a 'DRT-conditional-style' merge over info states:

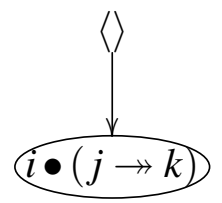

More details about IDPL are provided in Brasoveanu \& Dotlačil (2015c,b). Some highlights: we formalize DPropL models as extended monoids in the sense of Visser 2002; Vermeulen (1994) takes them to be Heyting algebras, but extended monoids 
Incremental and predictive interpretation

are more general (useful when we move to DPL), and more directly related to the relational models used in DPL, where formulas denote binary relations between variable assignments. The elements in the extended monoid are updates / formula denotations. The final form of the IDPL semantics follows Vermeulen 1994: it is a 'tree based' incremental semantics for DPropL and DPL: the tree encodes update histories, the nodes in the tree are updates / formula denotations, and conditionals are taken to introduce embedded / subordinate update histories along the lines of (26) above.

\subsection{An ACT-R based left-corner DRT parser}

Outlining our ACT-R based left-corner parser is beyond the scope of the present paper. We will only mention here the main idea, which is to use an independently motivated, general cognitive architecture (ACT-R, Anderson \& Lebiere 1998) with its theory of declarative memory on one hand, and procedural memory (cognitive skill) on the other, to give a mechanistic account of the specific task of simultaneous syntactic and semantic parsing. On the syntax side, we incrementally and predictively assemble a tree-like representation by incrementally constructing feature structures / attribute-value matrices - chunks, in ACT-R terms - of the kind used in HPSG (Pollard \& Sag 1994) a.o. On the semantics side, and in tandem with the syntactic parsing process, we incrementally and predictively assemble a DRT (Kamp \& Reyle 1993) representation for the meaning of a sentence.

Importantly, the proposal (and implementation) enables us to fully and explicitly integrate linguistic theory and experimental data, i.e., to provide integrated end-toend accounts of real-time language interpretation processes. These accounts link in an explicit way detailed, richly structured syntactic and formal semantics analyses on one end to accuracy and latency measures of the kind provided by psycholinguistic experiments at the other end. We are also able to make specific, fully formalized claims about processing complexity attributable to various factors, e.g., syntactic and/or semantic reanalysis, the recency of activation of particular syntactic and/or semantic representations, and the utility of the procedural rules used to incrementally and predictively construct such representations. Proof-of-concept parses of the cataphoric conditional in (7) above and its conjunction counterpart (John won't eat it and a hamburger is overcooked), as well as the full code for the current version of the ACT-R-based syntax-semantics parser are provided in Brasoveanu \& Dotlačil 2015a,b. 


\section{Conclusion}

To summarize, we used the interaction of cataphora and presupposition on one hand, and conjunctions vs. conditionals on the other, to provide evidence for the incremental and predictive nature of real-time meaning representation construction (the kind of meaning representations that are pervasive in formal semantics). We outlined two types of accounts: we can capture the incremental and predictive nature of real-time semantic interpretation in the semantics (Incremental Dynamic Predicate Logic, IDPL), or in the processor (an ACT-R based left-corner style DRT parser).

The issue of which (type of) account seems to be more suitable remains open. As far as the interpretation objects that are incrementally constructed, the two approaches are very similar: IDPL builds trees of info states incrementally, while the DRT parser ends up having to keep track of the accessibility relation between DRSs (needed for pronoun and cataphora resolution). There is no clear difference here except for the fact that the ACT-R based DRT parser automatically makes specific predictions about the time-course of interpretation (since it is a processing account). So as a theory, the DRT parser is more falsifiable but also less modular (it does not have a distinct computational-level theory of update-history trees), and possibly more brittle.

But when we examine the way in which anaphoric / cataphoric pronouns are resolved, there is a clear difference. IDPL (just like DPL) has nothing to say about this. Especially for cataphora, it is pretty clear that a goal to resolve the pronoun has to be separately maintained as the regular incremental interpretation process keeps marching forward, and the cataphoric goal is (repeatedly) retrieved during the incremental processing of the post-cataphoric text and its resolution is (repeatedly) attempted (see Kazanina et al. 2007 and Exp. 2 above for evidence). Thus, in IDPL, or any other purely semantic / competence account, a separate mechanism has to be postulated to keep track of this unresolved goal. In contrast, there is no need for additional mechanisms in a performance theory: the idea that high-level cognitive processes are goal driven is at the core of ACT-R and other cognitive architectures. But this difference is not necessarily an argument against IDPL, which is a 'competence', computational-level only theory of incremental interpretation. As such, it could be embedded in a variety of 'performance' theories.

It seems that both types of accounts need to be properly explored. And this necessity holds for both the principled and the strategic extensionalist positions. When we analyze phenomena in purely syntactic and/or semantic terms, we implicitly classify them as essentially belonging to the grammar, but this is ultimately an empirical, not a priori, decision, which even a principled extensionalist needs to face. We should probably consider alternative, processing based/laced explanations more systematically since it is hard to know at the outset what the best explanation 
Incremental and predictive interpretation

for a phenomenon is - compare the variety of accounts of NPI licensing proposed over the last 50 years or so (the mainly syntactic approach in Klima 1964, the semantic approach in Ladusaw 1979, the recent discussion of NPI processing effects in Vasishth, Bruüssow, Lewis \& Drenhaus 2008 a.o.). Thus, it is not always clear that a particular phenomenon should be accounted for in grammatical / competence or processing / performance terms, or as a mixture of both.

Enriching semantic evaluation contexts and providing a finer-grained recursive definition of truth and satisfaction for natural language interpretation (as in IDPL) might be the correct thing to do, but an independently needed theory of the real-time processing of semantic and syntactic representations (formulated in an independently motivated cognitive architecture) might also be able to account for crucial aspects of the phenomena under investigation. Or a 'hybrid', semantic and processing, approach might be the way to go. The only way to begin exploring this space of alternative explanations is to formulate mathematically explicit theories of how formal semantic representations are processed in real time and evaluate them empirically and theoretically.

\section{References}

Abusch, Dorit. 2010. Presupposition triggering from alternatives. Journal of Semantics 27. 37-80. doi:10.1093/jos/ffp009.

Anderson, John R. \& Christian Lebiere. 1998. The Atomic Components of Thought. Hillsdale, NJ: Lawrence Erlbaum Associates.

Barr, Dale J., Roger Levy, Christoph Scheepers \& Harry J. Tily. 2013. Random effects structure for confirmatory hypothesis testing: Keep it maximal. Journal of Memory and Language 68(3). 255-278. doi:10.1016/j.jml.2012.11.001.

Bates, Douglas, Martin Maechler, Ben Bolker \& Steven Walker. 2014. Ime4: Linear mixed-effects models using Eigen and S4. http://CRAN.R-project.org/package= lme4. R package version 1.1.7.

Bhatt, Rajesh \& Roumyana Pancheva. 2006. Conditionals. In Martin Everaert \& Henk van Riemsdijk (eds.), The Blackwell Companion to Syntax, 638-687. Wiley.

Brasoveanu, Adrian \& Jakub Dotlačil. 2015a. An ACT-R based left-corner style DRT parser: General design considerations and an implementation in Python ACT-R. UC Santa Cruz and University of Groningen ms., http://people.ucsc. edu/ abrsvn/ACT-R_based_DRT_parser.pdf.

Brasoveanu, Adrian \& Jakub Dotlačil. 2015b. Computing Dynamic Meanings Language, Cognition, and Mind (LCAM) Series. Dordrecht: Springer. In preparation.

Brasoveanu, Adrian \& Jakub Dotlačil. 2015c. Incremental interpretation and 
dynamic semantics. UC Santa Cruz and University of Groningen ms., http: //people.ucsc.edu/ abrsvn/inc_int_and_dyn_sem.pdf.

Chierchia, Gennaro. 1995. Dynamics of Meaning: Anaphora, Presupposition, and the Theory of Grammar. Chicago: University of Chicago Press.

Chomsky, Noam. 1965. Aspects of the Theory of Syntax. Cambridge, MA: MIT Press.

Clark, Herbert H. \& Barbara C. Malt. 1984. Psychological constraints on language: A commentary on Bresnan and Kaplan and Givón. In W. Kintsch, J.R. Miller \& P.G. Polson (eds.), Method and Tactics in Cognitive Science, 411-420. Hillsdale, NJ: Lawrence Erlbaum Associates.

Elbourne, Paul. 2009. Bishop sentences and donkey cataphora: A response to Barker and Shan. Semantics and Pragmatics 2. 1-7. doi:10.3765/sp.2.1.

Groenendijk, Jeroen \& Martin Stokhof. 1991. Dynamic predicate logic. Linguistics and Philosophy 14(1). 39-100. doi:10.1007/bf00628304.

Hagoort, Peter, Lea Hald, Marcel Bastiaansen \& Karl Magnus Petersson. 2004. Integration of word meaning and world knowledge in language comprehension. Science 304(5669). 438-441. doi:10.1126/science.1095455.

Hale, John. 2001. A probabilistic Earley parser as a psycholinguistic model. In The 2nd Meeting of the North American Asssociation for Computational Linguistics, 159-166.

Hale, John. 2011. What a rational parser would do. Cognitive Science 35. 399-443. Haubo Bojesen Christensen, Rune. 2013. ordinal—regression models for ordinal data. R package version 2013.9-30. http://cran.r-project.org/web/packages/ ordinal/index.html.

Heim, Irene. 1991. Artikel und definitheit. In A. von Stechow \& D. Wunderlich (eds.), Semantik: Ein internationales Handbuch der zeitgenössischen Forschung, 40-64. Berlin: Walter de Gruyter.

Hofmeister, Philip, Laura Staum Casasanto \& Ivan A. Sag. 2013. Islands in the grammar? Standards of evidence. In Jon Sprouse \& Norbert Hornstein (eds.), Experimental Syntax and Island Effects, 42-63. Cambridge University Press.

Hough, Julian, Casey Kennington, David Schlangen \& Jonathan Ginzburg. 2015. Incremental semantics for dialogue processing: Requirements and a comparison of two approaches. In The International Workshop on Computational Semantics (IWCS) 2015, .

Iatridou, Sabine. 1991. Topics in conditionals: MIT PhD dissertation.

Just, Marcel A., Patricia A. Carpenter \& Jacqueline D. Woolley. 1982. Paradigms and processes in reading comprehension. Journal of Experimental Psychology: General 111(2). 228-238. doi:10.1037/0096-3445.111.2.228.

Kamp, Hans. 1981. A theory of truth and semantic representation. In Jeroen Groenendijk, Theo Janssen \& Martin Stokhof (eds.), Formal Methods in the 
Incremental and predictive interpretation

Study of Language, 277-322. Amsterdam: Mathematical Centre Tracts.

Kamp, Hans \& Uwe Reyle. 1993. From Discourse to Logic: Introduction to Modeltheoretic Semantics of Natural Language, Formal Logic and Discourse Representation Theory. Dordrecht: Kluwer.

Kazanina, Nina, Ellen F. Lau, Moti Lieberman, Masaya Yoshida \& Colin Phillips. 2007. The effect of syntactic constraints on the processing of backwards anaphora. Journal of Memory and Language 56. 384-409. doi:10.1016/j.jml.2006.09.003.

Klima, Edward. 1964. Negation in English. In Jerry A. Fodor \& Jerrold J. Katz (eds.), The Structure of Language: Readings in the Philosophy of Language, 246-323. Prentice-Hall, Englewood Cliffs.

Kuznetsova, Alexandra, Per Bruun Brockhoff \& Rune Haubo Bojesen Christensen. 2014. lmerTest: Tests for random and fixed effects for linear mixed effect models (lmer objects of lme4 package). http://CRAN.R-project.org/package=lmerTest. $\mathrm{R}$ package version 2.0.11.

Ladusaw, William. 1979. Polarity sensitivity as inherent scope relations: University of Texas $\mathrm{PhD}$ dissertation.

Lau, Ellen F. 2009. The Predictive Nature of Language Comprehension: University of Maryland, College Park PhD dissertation.

Levy, Roger. 2008. Expectation-based syntactic comprehension. Cognition 106. 1126-1177. doi:10.1016/j.cognition.2007.05.006.

Lewis, Richard \& Shravan Vasishth. 2005. An activation-based model of sentence processing as skilled memory retrieval. Cognitive Science 29. 1-45. doi:10.1207/s15516709cog0000_25.

Marr, David. 1982. Vision: A computational investigation into the human representation and processing of visual information. San Francisco: W. H. Freeman and Company.

Phillips, Colin. 1996. Order and structure: Massachusetts Institute of Technology $\mathrm{PhD}$ dissertation.

Phillips, Colin. 2003. Linear order and constituency. Linguistic Inquiry 34(1). 37-90. doi:10.1162/002438903763255922.

Phillips, Colin \& Shevaun Lewis. 2013. Derivational order in syntax: evidence and architectural consequences. Studies in Linguistics 6. 11-47.

Pickering, Martin J, Brian McElree, Steven Frisson, Lillian Chen \& Matthew J Traxler. 2006. Underspecification and aspectual coercion. Discourse Processes 42(2). 131-155. doi:10.1207/s15326950dp4202_3.

Poesio, Massimo. 1994. Ambiguity, underspecification and discourse interpretation. In The First International Workshop on Computational Semantics, 151-160.

Pollard, Carl \& Ivan A. Sag. 1994. Head-driven phrase structure grammar. University of Chicago Press. 
R Core Team. 2014. R: A Language and Environment for Statistical Computing. R Foundation for Statistical Computing Vienna, Austria. http://www.R-project. org/.

de Saussure, Ferdinand. 1916. Cours de Linguistique Générale. Payot. Reprinted in 1995.

Schwarz, Florian. 2014. Presuppositions are fast, whether hard or soft - evidence from the visual world. In Todd Snider, Sarah D'Antonio \& Mia Wiegand (eds.), Semantics and Linguistic Theory (SALT), vol. 24, 1-22. LSA and CLC Publications.

Steedman, Mark. 2001. The Syntactic Process. Cambridge, MA: MIT Press.

Stewart, Terrence C. 2007. A Methodology for Computational Cognitive Modelling. Ottawa, Ontario: Carleton University PhD dissertation.

Stewart, Terrence C. \& Robert L. West. 2007. Deconstructing and reconstructing ACT-R: Exploring the architectural space. Cognitive Systems Research 8. 227236. doi:10.1016/j.cogsys.2007.06.006.

Stowe, Laurie A. 1986. Parsing wh-constructions: Evidence for on-line gap location. Language and Cognitive Processes 1(3). 227-245.

Traxler, Matthew J. \& Martin J. Pickering. 1996. Plausibility and the processing of unbounded dependencies: An eye-tracking study. Journal of Memory and Language 35(3). 454-475. doi:10.1006/jmla.1996.0025.

Trueswell, John, Michael Tanenhaus \& Susan Garnsey. 1994. Semantic influences on parsing: Use of thematic role information in syntactic ambiguity resolution. Journal of Memory and Language 33(3). 285-318. doi:10.1006/jmla.1994.1014.

Vasishth, Shravan, Sven Bruüssow, Richar L. Lewis \& Heiner Drenhaus. 2008. Processing polarity: How the ungrammatical intrudes on the grammatical. Cognitive Science 32. 685-712.

Vermeulen, C.F.M. 1994. Incremental semantics for propositional texts. Notre Dame Journal of Formal Logic 35(2). 243-271. doi:10.1305/ndjfl/1094061863.

Visser, Albert. 2002. The donkey and the monoid. Journal of Logic, Language and Information 11. 107-131. doi:10.1023/a:1013026830239.

Wagers, Matthew W. \& Colin Phillips. 2009. Multiple dependencies and the role of the grammar in real-time comprehension. Journal of Linguistics 45. 395-433. doi:10.1017/s0022226709005726.

Wickham, Hadley. 2009. ggplot2: elegant graphics for data analysis. New York: Springer. 
Incremental and predictive interpretation

Adrian Brasoveanu

Jakub Dotlačil

Linguistics, UC Santa Cruz

1156 High Street

Santa Cruz, CA 95064, USA

University of Groningen

Algemene taalwetenschap

abrsvn@\{gmail.com,ucsc.edu

Oude Kijk in 't Jatstraat 26

9712 EK Groningen

The Netherlands

j.dotlacil@gmail.com 